\title{
Renal Disease
}

\author{
Priyanka Sehrawat ${ }^{1}$, Pranav Bansal ${ }^{2}$ \\ 1. Resident; 2. Professor\& Head, Department of Anesthesiology \& Critical Care, BPS Govt Medical College, Khanpur Kalan, \\ Sonepat, Haryana, India.
}

Correspondence: Professor Pranav Bansal; E-mail: pranavbansal1@gmail.com; Phone: +91-8222007555

Q 1. Which of the following statement is false with respect to renal blood flow:-

a. Kidneys receive approximately $15-20 \%$ of total cardiac output

b. Renal papillae are most vulnerable to ischemic insults as it receives only $5 \%$ of the cardiac output

c. Glomerular capillaries are low pressure system whereas peritubular capillaries are high pressure systems

d. Anesthetic agents does not have direct impact on renal blood flow, alterations occur secondary to changes in cardiac output

Q 2. Conditions that increases Blood Urea Nitrogen independently of glomerular filtration rate except:-
a. Corticosteroid use
b. Sickle cell anemia
c. High protein diet
d. Antibiotics such as Tetracycline

Q 3. According to management guidelines for correction of anemia of chronic renal disease, which statement is correct:-
a. Target hemoglobin is $8-10 \mathrm{~g} / \mathrm{dl}$
b. Iron therapy should be withhold when percent transferrin saturation $>80 \%$
c. Erythropoietin and darbepotin ALFA should only be administered weekly
d. Optimal rate of correction is increase in hemoglobin by $1-2 \mathrm{~g} / \mathrm{dl}$ over 4 week period

Q 4. Which of the following statement is false regarding uremic bleeding:

a. Bleeding risk correlates well with serum BUN

b. Patients usually have normal platelet count/ normal PT / normal INR

c. Bleeding time is the best screening test

d. Desmopressin acts by increasing levels of factor VIII - vWF complex.

Q 5. Regarding the classification system for acute renal failure, which statement is not correct:-

a. The $F$ in RIFLE stands for failure and corresponds to stage 3 of AKIN class b. Kidney failure cannot be diagnosed based on urine output alone.

c. Criteria for risk is either elevation creatinine $>1.5 \times$ normal or urine output $<0.5 \mathrm{ml} / \mathrm{kg} / \mathrm{h}$ for $6 \mathrm{~h}$ or fall in GFR $>25 \%$

d. Patients receiving renal replacement therapy falls under stage 3 of AKIN

Q 6. Which statement is false regarding fractional excretion of sodium:-

a. Differentiate between prerenal and renal azotemia

b. It is the filtered sodium divided by GFR

c. $\mathrm{FE}_{\mathrm{Na}}<2 \%$ reflects decreased ability of renal tubules to conserve sodium

d. Reflects tubular function

Q 7. False statement with respect to drug dosing in a patient with renal impairment is:-

a. Renal failure may impair absorption, distribution, metabolism and excretion of a drug

b. Done by calculating the GFR

c. In oliguric patients, the creatinine clearance can be approximated by a value of $5 \mathrm{ml} / \mathrm{min}$

d. The loading dose of a drug to rapidly achieve therapeutic levels needs to modified irrespective of the extracellular fluid status

Q 8. True statement regarding intradialytic complications is:-

a. Hypotension is the most common adverse effect and successfully treated by administering iv saline

b. Hypersensitivity reactions to ethylene oxide are extremely rare

c. Dialysis disequilibrium syndrome is benign and marked by nausea and fatigue only

d. Reactions to polyacrylonitrile are rarely seen with patients on ACE inhibitors

Q 9. A 52 year old male, known chronic alcoholic, with bilateral pitting edema and abdominal distension recently developed oliguria, which of the following statement is incorrect for his condition:-

a. Hypoalbuminemia and hypoprothrombinemia are present 
b. Severe renal arterial and arteriolar vasoconstriction often leads to such condition

c. Volume replacement and splanchnic vasoconstriction by vasopressin receptor agonists may help in improving renal perfusion and GFR

d. Peritoneal- venous shunt adds no improvement in renal function

Q 10. All of the following mentioned statements regarding peritoneal dialysis are true except:

a. Survival rates are lower with peritoneal dialysis

b. May be desirable for patients with $\mathrm{CHF}$ or unstable angina

c. Presence of abdominal hernias and adhesions may interfere with the ability to use peritoneal dialysis effectively

d. Peritonitis is encountered as most serious complication

\section{Explanations:}

Ans 1(c): The kidneys receive approximately $15 \%$ to $25 \%$ of total cardiac output, or 1 to $1.25 \mathrm{~L} / \mathrm{min}$ of blood, through the renal arteries, depending on the state of the body. Most of the blood is received by the renal cortex, with only $5 \%$ of cardiac output flowing through the renal medulla, which makes the renal papillae vulnerable to ischemic insults. Glomerular capillaries are highpressure systems, whereas peritubular capillaries are low-pressure systems. Sympathetic stimulation resulting from surgery can increase vascular resistance and reduce renal blood flow, whereas anesthetics may reduce renal blood flow by decreasing cardiac output.

Ans (b): Conditions causing increase in BUN

- Catabolic state (steroid usage, GI bleed)

- Reduced effective circulatory volume

- High protein diet

- Tetracycline

Conditions causing decrease in BUN

- Liver disease

- Sickle cell anemia

- SIADH

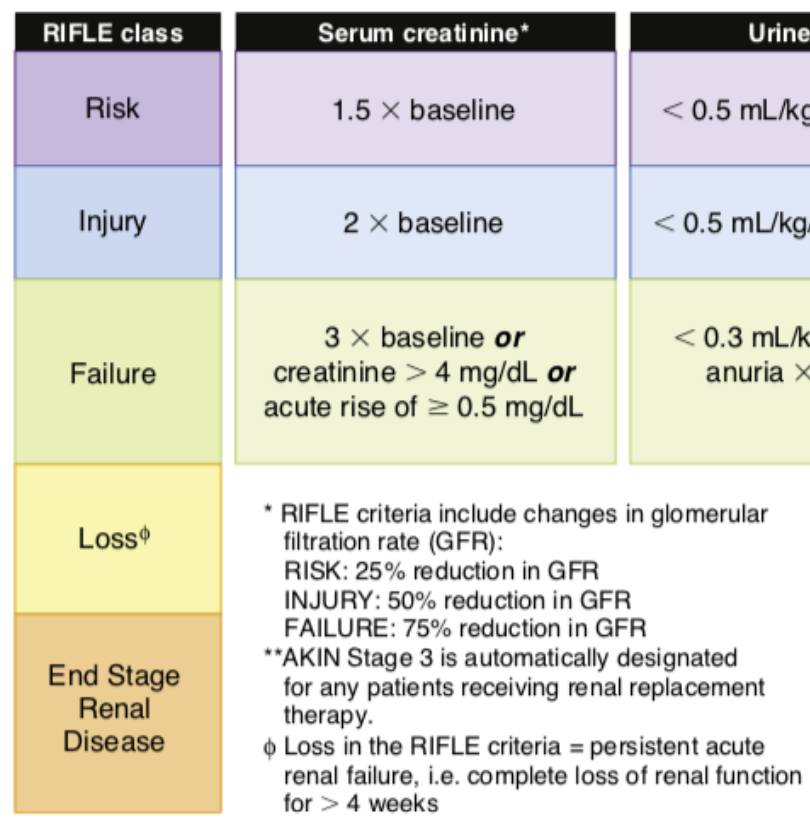

- Malnutrition

Ans 3 (d): Withhold iron therapy when Tsat $>50 \%$ or ferritin $>800 \mathrm{ng} / \mathrm{ml}$

Target hemoglobin is $11-12 \mathrm{~g} / \mathrm{dl}$

Erythropoietin can be administered once, twice or three times per week if required; whereas darbepoetin ALPA is administered single injection IV/subcutaneous once weekly or every 2 weeks.

Ans 4 (a): Patients with CKD have an increased tendency to bleed despite the presence of a normal platelet count, prothrombin time, and plasma thromboplastin time. The bleeding time is the screening test that best correlates with the tendency to bleed. Treatment of uremic bleeding may include the administration of cryoprecipitate to provide factor VIIIvWF complex or the use of desmopressin. Desmopressin, an analogue of antidiuretic hormone, increases circulating levels of factor VIII-vWF complex and thereby improves coagulation.

Ans 5 (b)
Urine output

$3 \mathrm{~mL} / \mathrm{kg} / \mathrm{hr} \times 24$ or

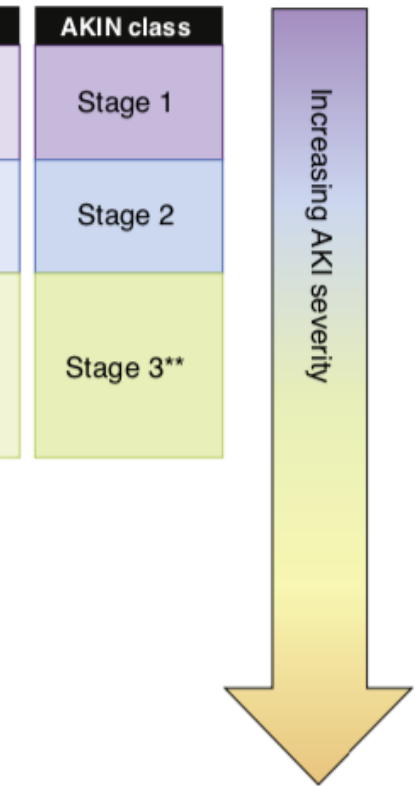


Ans 6 (c): The fractional excretion of sodium $\left(\mathrm{FeNa}_{\mathrm{Na}}\right)$ is a measure of the percentage of filtered sodium that is excreted in the urine. It is most useful in differentiating between pre- renal and renal causes of azotemia. $\mathrm{Fe}_{\mathrm{Na}}$ higher than $2 \%$ (or urinary sodium concentration of $>40$ $\mathrm{mEq} / \mathrm{L}$ ) reflects decreased ability of the renal tubules to conserve sodium and is consistent with tubular dysfunction.

Ans 7(d): The first step in drug dosing for patients with renal impairment is to estimate the creatinine clearance, since the rate of elimination of drugs excreted by the kidneys is proportional to the GFR. If the patient is oliguric, the creatinine clearance can be approximated by a value of $5 \mathrm{ml} / \mathrm{min}$. If after clinical examination, the extracellular fluid volume appears to be normal, use the loading dose suggested for patients with normal renal function. If the extracellular fluid is contracted, reduce the loading dose. If the extracellular fluid is expanded, use a higher loading dose.

Ans 8 (a): Intradialytic complications: Hypotension is the most common adverse event during hemodialysis and most likely reflects osmolar shifts and ultrafiltrationinduced volume depletion. Hypersensitivity reactions to the ethylene oxide used to sterilize dialysis machines, as well as adverse reactions to the specific hemodialysis membrane material polyacrylonitrile, may occur. Reactions to polyacrylonitrile are seen most commonly in patients receiving ACE inhibitors. Dialysis disequilibrium syndrome is marked by nausea, headaches, and fatigue, but may progress to seizures or coma. The condition results from rapid changes in $\mathrm{pH}$ and solute concentrations in the central nervous system

Ans 9 (d): Acute oliguria manifesting in patients with decompensated cirrhosis of the liver is called hepatorenal syndrome. The typical patient is deeply jaundiced and moribund; ascites, hypoalbuminemia, and hypoprothrombinemia are present. Renal failure in these patients reflects reduction in effective circulating volume, partly as a result of diuretic treatment and partly as a result of splanchnic arteriolar dilatation. Treatment is directed at restoring intravascular fluid volume. Type 1 is more serious and accompany rapid decline in renal function.

Ans 10 (a): Survival rates and annual costs are similar with peritoneal dialysis and hemodialysis, but hospitalization rates are higher among patients treated with peritoneal dialysis. peritoneal dialysis may be desirable for patients with congestive heart failure or unstable angina who may not tolerate the rapid fluid shifts or fluctuations in systemic blood pressure that often accompany hemodialysis. Peritonitis presenting as abdominal pain and fever is the most common serious complication of peritoneal dialysis. Treatment is with antibiotics, which may include cephalosporins, aminoglycosides, and vancomycin. 